\title{
隔离基团修饰的有机二阶非线性光学发色团
}

\author{
陈 璐 $a, b$ 薄淑晖*, $a$ 和延岭 $a, b$ 陈 卓 $a$ \\ 刘新厚 $a$ 甄珍*, $a$ \\ ( ${ }^{a}$ 中国科学院理化技术研究所 光化学转换与功能材料重点实验室 北京 100190) \\ $\left({ }^{b}\right.$ 中国科学院大学 北京 100043)
}

\begin{abstract}
摘要 有机二阶非线性光学发色团是非线性光学材料的重要组成部分. 一直以来, 科学家们致力于通过结构设计与修 饰来研究合成新型的性能良好的发色团. 然而如何将有机二阶非线性光学发色团微观一阶超极化率 $\beta$ 值高效地转化为 宏观电光系数 $r_{33}$, 一直是极具挑战性的课题. 近几年来, 有诸多报道通过引入一些隔离基团来改变发色团的形状, 可 以有效地减少极化过程中分子之间的偶极相互作用力, 有利于实现大的宏观电光系数 $r_{33}$ 值. 结合本课题组的研究, 系 统总结了近些年有关在发色团中引入一些修饰隔离基团的研究工作.
\end{abstract}

关键词 有机二阶非线性光学材料; 发色团; 隔离基团; 电光活性; 偶极-偶极相互作用

\section{Organic Second-Order Nonlinear Optical Chromophores Modified by Isolation Groups}

\author{
Chen, $\mathrm{Lu}^{a, b}$ \\ Bo, Shuhui*,a \\ He, Yanling ${ }^{a, b}$ \\ Chen, Zhuo ${ }^{a}$ \\ Liu, Xinhou ${ }^{a}$ \\ Zhen, Zhen *,a
}

( ${ }^{a}$ Key Laboratory of Photochemical Conversion and Optoelectronic Materials, Technical Institute of Physics and Chemistry, Chinese Academy of Sciences, Beijing 100190)

( ${ }^{b}$ University of Chinese Academy of Sciences, Beijing 100043)

\begin{abstract}
Organic second-order nonlinear optical (NLO) chromophores are the main constituent part of nonlinear optical material. Scientists are dedicated to researching on the synthesis of novel chromophores with excellent performance by the facile design and modification of organic second-order NLO chromophores for decades. However, the researches on NLO chromophores in order to achieve the efficient translation of molecular microscopic nonlinearity (hyperpolarizability $\beta$ ) into macroscopic electro-optic (EO) coefficients $r_{33}$ is still a challenging task. In recent years, a large number of essays report the novel NLO chromophores by adding isolation groups which can change the shape of chromophores and decrease the electrostatic interaction aimed to achieve the bigger EO coefficients. In this review, the recent processes of novel NLO chromophores modified by isolation groups are reviewed systematically and comprehensively.
\end{abstract}

Keywords organic second-order nonlinear optical materials; chromospheres; isolation group; electro-optic activity; dipole-dipole interaction

由于信息通讯技术的高速发展, 以电子作为载体的 信息传输已经无法满足现代人们对于高速数据传输、处 理和大容量信息运算的需求. 相比较以电子作为信息载 体, 光子作为信息载体具有良好并行性、高频率、宽带 宽、高速度以及抗电磁波干扰等优点, 成为未来发展的 必然趋势 ${ }^{[1]}$. 集成光系统融合了电子技术和光学技术, 包含了波导、光纤、光开关 ${ }^{[2]}$ 、电光调制器等重要元器
件 ${ }^{[3]}$, 而非线性光学材料在研究这些器件中发挥了重要 作用 ${ }^{[4,5 \mathrm{a}, 5 \mathrm{~b}]}$.

非线性光学(Nonlinear Optic, 简称 NLO)是研究在 强光(激光)的作用下物质的响应与场强呈现非线性关系 的一门科学, 与场强有关的光学效应称为非线性光学效 应. 在光学理论中, 通常采用光在介质中引起的极化响 应来描述光与介质的相互作用以及光在介质中的传播

\footnotetext{
* Corresponding authors. E-mail: zhenzhen@mail.ipc.ac.cn, boshuhui@mail.ipc.ac.cn Received March 2, 2017; revised April 28, 2017; published online May 17, 2017. Project supported by the National Natural Science Foundation of China (No. 21504099). 国家自然科学基金(No. 21504099)资助项目..
} 
特性. 当光在介质中传播时, 介质中的价电子在光电场 作用下产生相对于原子的电荷转移, 引起介质极化. 其 极化强度与入射光的光电场强度 $E$ 的关系为 ${ }^{[6]}$ :

$$
\begin{aligned}
& \rho=\alpha E+\beta E E+\gamma E E E+\cdots \\
& P=\varepsilon_{0} \chi^{(1)} E+\varepsilon_{0} \chi^{(2)} E E+\varepsilon_{0} \chi^{(3)} E E E+\cdots
\end{aligned}
$$

在 Eq. 1 中 $\rho$ 表示微观分子在外加电场作用下的极化强 度. $\alpha, \beta, \gamma$ 分别为分子的线性极化率、一阶超极化率和二 阶超极化率. 在 Eq. 2 中 $P$ 表示材料的宏观极化强度, $\varepsilon_{0}$ 表示真空介电常数, $\chi^{(1)}$ 是一阶线性极化率, $\chi^{(2)}$ 是二阶非 线性极化率, $\chi^{(3)}$ 是三阶非线性极化率, 依次类推. 在外 加电场比较弱的情况下, Eqs. 1, 2 中等号右边从第二项 起可以忽略, 此时感应极化强度与电场强度 $(E)$ 为线性 关系: $\rho=\alpha E, P=\varepsilon_{0} \chi^{(1)}$ 属于线性光学范畴. 而在激光的 作用下，上述两个公式中的第二项乃至以后各项就不能 忽略, 这是由于激光是相干高强光, 具有与原子束缚内 电场可以相比较的电磁场. 因此导致感应极化强度 $\rho$ 和 $P$ 与光电场强度为非线性关系. 其中与第 2 项有关的效 应称为二阶(second-order)非线性光学效应, 与第 3 项有 关的效应称为三阶(third-order)非线性光学效应. 关系式 中的奇数项系数不为零, 而对于偶数项系数 (如 $\beta$ 和 $\chi^{(2)}$ ), 只有那些非中心对称的介质才不为零, 才有可能观测到 相应的非线性光学效应. 这就决定了只有宏观上表现非 中心对称结构特征的偶极材料才能表现出二阶非线性 光学效应 ${ }^{[7]}$.

电光效应(electro-optic effect)是一种典型的非线性 光学效应, 它是指在外加电场(直流或射频电场)的作用 下, 光学介质的折射率 $n$ 发生变化的现象. 若外加电场 引起的折射率变化正比与电场强度 $E$, 即 $n$ 仅与 $E$ 的一 次项相关, 称为线性(一次)电光效应, 也称为 Pockels 效 应, 属于二阶非线性光学效应. 只有那些不具有反演对 称性的材料才能产生 Pockels 效应, 所谓无反演对称性 即材料无对称中心. 在线性电光效应中, 材料折射率的 变化 $\Delta n$ 与外加电场的关系如下:

$$
\Delta n=n_{0}{ }^{3} r E / 2
$$

其中 $r$ 为线性电光系数, 简称电光系数 (Electro-Optic coefficient), 单位 $\mathrm{pm} / \mathrm{V}$, 是衡量宏观电光效应强弱的物 理量. 从本质上而言, 线性电光效应是在光波电场和外 加电场的共同作用下, 引起材料的非线性极化, 并导致 其光学各向异性, 因而线性电光效应实际上是二阶非线 性光学现象的一种特殊情况, 要求构成材料的分子集合 体必须具有非中心对称的结构.

二阶非线性光学材料按照化学组成体系大致可分 为无机材料、有机材料和有机/无机杂化材料三大类 ${ }^{[8 a, b]}$. 其中有机材料相比较无机材料具有二阶非线性光学系

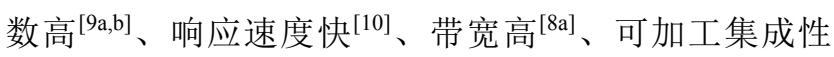
好 $^{[11]}$ 、选择范围广 ${ }^{[12]}$ 、介电常数低 ${ }^{[13]}$ 以及相位失配小 ${ }^{[14]}$ 等优点. 有机非线性光学材料的这些优势使其成为新型 光电子器件组成材料的研究热点. 然而实际应用和生产 中还需要克服一些技术和工艺问题: (1)更高的材料电光 系数 $r_{33}$; (2) 更小的半波电压; (3)良好的稳定性(化学, 物 理, 取向); (4)简化器件制备工艺和加工技术.

波导开关、调制器、滤光器、偏振转换器等波导器 件是利用非线性光学材料的线性电光效应而制造的. 电 光调制器的制备涉及诸多交叉的学科领域如光学工程、 高分子化学、有机化学以及材料器件加工工程等. 常用 的调制器类型包括 Mach-Zehnder 干涉电光调制器(M$Z$ )、电吸收调制器(EA)、双向耦合器、双折射调制器等. 本课题组长期从事有机 Mach-Zehnder 干涉电光调制器 的研究工作. 图 1,2 是目前已经商品化的电光调制器的 构造, 分别为电光调制器的三维构造和电光调制水平剖 面图, 即含有电光材料的核心波导光路 ${ }^{[15]}$.

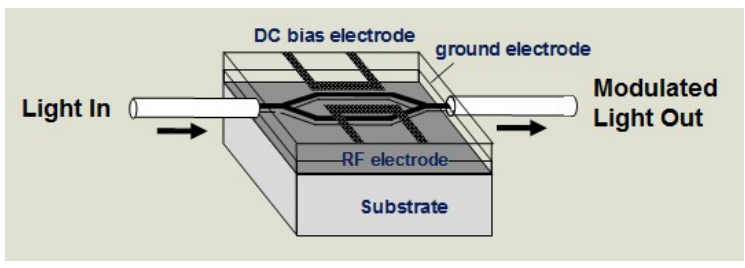

图 1 电光调制器的三维构造 $[15 \mathrm{~b}, 15 \mathrm{c}]$

Figure 1 Three-dimensional structure of electro-optic modulator $^{[15 b, 15 c]}$

有机二阶非线性光学发色团(有机电光发色团)是有

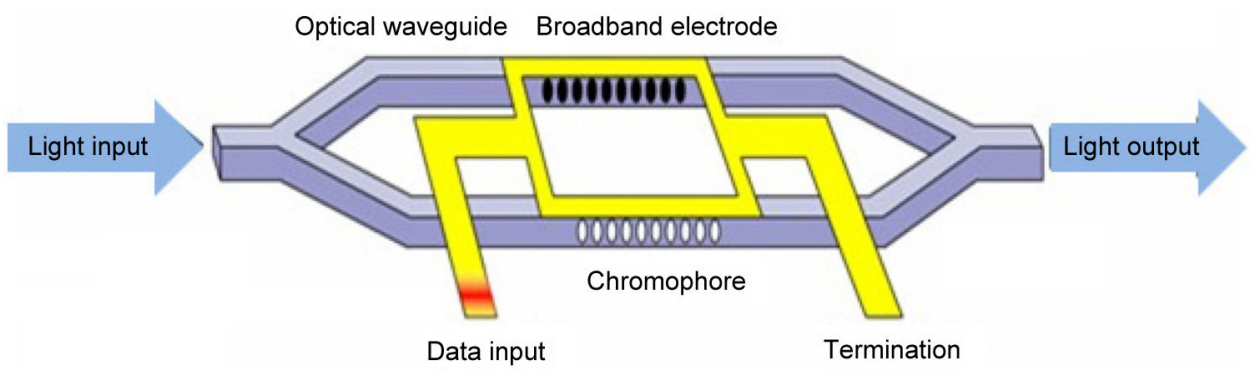

图 2 电光调制水平剖面图 ${ }^{[15 b, c]}$

Figure 2 Horizontal section of electro-optic modulator ${ }^{[15 b, 15 c]}$ 
机二阶非线性光学材料(有机电光材料)的核心组成部 分, 它一般是由电子给体(electron donor)和电子受体 (electron acceptor)及共轭 $\pi$ 电子桥( $\pi$-electron bridge)组 成. 如图 3 所示.

\section{电子给体 $\mathrm{D} \square$ 共轭 $\pi$ 电子桥}

图 $3 \mathrm{D}-\pi-\mathrm{A}$ 分子的结构组成

Figure 3 Structure of D- $\pi$-A molecule

这种推拉型(Push-Pull)偶极发色团分子也被称为 $\mathrm{D}-\pi-\mathrm{A}$ 分子, 分子的一阶超极化率 $\beta$ 常常用于衡量分子 的微观非线性 ${ }^{[16]}$, 主要由发色团的结构、电子效应等因 素决定. 发色团的电子给体具有强给电子能力, 通常包 含孤对电子的杂原子如 $\mathrm{N} 、 \mathrm{O} 、 \mathrm{~S}$ 等, 能够增加分子内 $\pi$ 电子的密度, 增强电子流动性, 从而有利于 $\beta$ 值的增加; 电子受体一般是由强吸电子能力基团组成; 而共轭 $\pi$ 电 子桥是连接给体和受体的部分, 则要求具有很好的电子 迁移能力, 这三部分共同组成了发色团 D- $\pi-\mathrm{A}$ 型的分子 结构 ${ }^{[17]}$.

发色团分子形状类似于棒状，具有一定的平面性， 因此在聚合物体系中会存在分子与分子之间较强的偶 极-偶极相互作用力, 这会影响极化过程中非中心对称 分子的取向排列 ${ }^{[18]}$. 此外, 如果在材料中增加有机非线 性发色团的浓度，分子间的间距就会减少，分子之间的 相互作用力增强, 同时还会出现主客体相分离, 导致光 学损耗, 所以实际宏观材料的电光活性 $r_{33}$ 值远低于理 论计算值. 如何减少分子间的偶极相互作用力以及更好 地将发色团的微观一阶超极化率 $\beta$ 值转化成材料的宏观 电光活性 $r_{33}$ 值, 已经成为研究电光材料需要克服的热 点问题 ${ }^{[19,20]}$. 近 10 多年来, 科学家们一直致力于优化分 子结构, 提高分子的微观非线性的同时能更好地转换成 宏观 $r_{33}$ 值, 为研制性能优越的器件奠定基础 ${ }^{[21]}$. 利用 隔离基团对现有的优良给体、受体、共轭桥进行修饰, 成 为目前解决偶极相互作用提高宏观电光系数的最常用 且行之有效的方法 ${ }^{[22]}$. 隔离基团是在发色团主体 D- $\pi-A$ 结构之外具有一定空间位阻的基团. 该基团具有一定的 位阻效应, 能够减弱发色团之间的静电相互作用, 有利 于发色团的转动取向 ${ }^{[23]}$.

本课题自 2004 年以来, 一直专注于有机非线性光 学材料的研究, 本文结合本课题组以及国内外其他课题 组的研究工作, 较为系统地介绍在有机电光发色团分子 中引入隔离基团的研究进展.

\section{1 隔离基团相关理论}

1999 年, 华盛顿大学 Dalton 课题组 ${ }^{[24]}$ 提出了 “isolation group” 观点, 通过对发色团分子进行修饰, 引
入一些隔离基团，减少分子之间的偶极相互作用，从而 提高材料的光学性能. 此理论的提出大大推动了有机电 光材料的发展, 后来陆续有文章报道在发色团分子的结 构中引入个性化修饰的隔离基团, 比如柔性长链的隔离 基团 ${ }^{[21 b]}$ 、末端连接官能团的隔离基团 ${ }^{[25]}$ 、刚性大位阻 隔离基团 ${ }^{[26]}$, 具有给电子杂原子的隔离基团等 ${ }^{[27]}$. 个性 化修饰的隔离基团一方面能够增加分子之间的距离, 减 少分子间的静电相互作用，从而提高材料的电光性能; 另一方面针对不同分子结构的发色团，个性化的修饰， 也可以提高分子的其他方面的性能, 比如溶解度、稳定 性等 ${ }^{[28]}$.

具有推拉型电子结构的发色团分子在极化时会产 生分子间的静电作用，从而造成分子的堆积 ${ }^{[29]}$, 隔离基 团的引入虽然能够降低分子之间的相互作用力, 减少分 子堆积, 然而也会直接影响发色团在高聚物中的浓度, 因此, 需要一种适合的隔离基团来平衡这两种因素产生 的影响. 武汉大学李振课题组在 “isolation group” 理论 基础上提出 “suitable isolation group” 的理论 ${ }^{[22,30]}$. 该理 论认为对于一个特定的发色团分子存在能够将分子之 间的偶极相互作用降到最低的合适隔离基团, 从而使得 分子的微观一阶超极化率 $\beta$ 值高效地转化为聚合物电光 材料的宏观 $r_{33}$ 或 $d_{33}$ 值 ${ }^{[31]}$.

\section{2 以隔离基团为修饰的发色团}

由给体、共轭桥、受体构成的具有电子推拉结构的 发色团分子是有机二阶非线性光学材料的核心组成部 分, 这三部分的结构决定了材料的电光活性. 因此, 科 学家们主要采取以下 4 种方法来设计并合成具有较高电 光性能的新型发色团: (1)引入具有较强给电子能力的新 型给体; (2)引入具有较强吸电子能力的新型受体; (3)引 入较强传递电子能力的共轭桥; (4)引入隔离基团, 其中 包括柔性链及刚性隔离基团. 前三种方法旨在增强发色 团分子中电子流动的密度. 第四种设计方法则是利用隔 离基团增大分子之间的间隔，减少极化过程中的静电相 互作用．隔离基团可以修饰发色团给体、共轭桥以及电 子受体部分, 但由于受体种类较少，所以隔离基团修饰 发色团的给体和共轭桥部分的研究相对比较多. 柔性隔 离基团修饰发色团，一方面能够增大分子之间的距离, 减少极化过程中产生的静电作用; 另一方面能够有效提 高发色团的溶解性能. 刚性隔离基团一般是由具有较大 空间位阻的刚性基团如苯环等组成，除了能够大大减少 分子之间的堆积, 提高微观一阶超极化率 $\beta$ 值向宏观电 光材料 $r_{33}$ 值的转化效率外, 还能够增强发色团的热稳 定性. 后文将分别介绍在发色团给体和电子桥上引入隔 离基团对发色团的性能所产生的影响. 


\section{1 隔离基团修饰发色团给体}

苯胺由于其 $\mathrm{N}$ 原子的给电子能力较强、平面性较好, 故一直是比较好的给体, 迄今为止有不少文章报道以苯 胺作为给体的发色团. 其中华盛顿大学 Dalton 课题 组 ${ }^{[21 a, 32]}$ 合成的经典发色团 FTC, 其给体就是苯胺. 较好 的平面性能虽然有利于分子内电子的流动, 但是在极化 过程中, 较好的平面性能也会增加发色团分子之间的偶 极-偶极相互作用的概率, 从而不利于分子的取向排 列 ${ }^{[33]}$. 因此, 理论计算的一阶微观超极化率 $\beta$ 值较高, 而材料的宏观电光系数 $r_{33}$ 值却较小, 为此不少研究者 对其结构进行改进和修饰, 方法总结如下: (1)在 FTC 发 色团的给体或共轭电子桥中增加一些隔离基团, 降低发 色团之间的偶极作用，提高电光材料的宏观电光性能; (2)利用隔离基团的可修饰性, 引入刚性大位阻基团提 高 FTC 发色团的热稳定性; (3)引入柔性支链, 提高其溶 解性能等.

本课题组以 FTC 发色团(烷基苯胺给体噻吩并多烯 共轭电子桥三氰基呋喃受体发色团)为对比, 保留了 FTC 分子中共轭桥和受体结构, 引入新型电子给体, 同 时在新型给体结构上引入具有一定给电子能力的刚性 隔离基团 ${ }^{[34,35]}$, 合成发色团 $\mathbf{1}$ 和 2 . 发色团主要的合成方 法相类似, 现以发色团 $\mathbf{1}$ 的合成为例, 总结了隔离基团 修饰的发色团的合成方法, 为日后科研工作者们关于隔 离基团修饰的有机二阶非线性光学发色团的合成方法 和策略部分的讨论提供借鉴. 主要合成步骤为以下 4 步: (1)经隔离基团修饰的给体首先经过 Vilsmeier 甲酰化反 应生成醛基; (2)经过 Witting 缩合反应, 将发色团的给 体和共轭桥通过一个碳碳双键相连接; (3) 利用 $n$ - BuLi 在一 $78{ }^{\circ} \mathrm{C}$ 拔掉给体一共轭桥中的氢, 再与 $N, N$-二甲基甲 酰胺(DMF)反应生成醛基(一般噻吩桥是用此方法接醛 基, 若电子桥为异佛尔酮桥, 则用 Vilsmeier 甲酰化反应 接醛基); (4)最后一步利用 Knoevenagel 缩合反应将给 体一电子桥与电子受体通过双键相连接.

对含有这三种发色团的电光材料的电光活性进行 测试, 最后实际所测的 FTC、分子 $\mathbf{1}$ 及分子 $\mathbf{2}$ 的 $r_{33}$ 值分 别为 $39,57,100 \mathrm{pm} / \mathrm{V}$. 分子 $\mathbf{1}$ 结构与 FTC 结构相比(图 4), 在苯胺的基础上引入具有一定位阻效应的饱和六元 环, 增大了发色团分子之间的距离, 削弱了极化时分子 之间的偶极相互作用; 另外将给体 $\mathrm{N}$ 原子所连接的一个 短链烷乙基改为一个柔性中长链烷丁基, 除增大位阻效 应外, 还有利于提高发色团的溶解度. 分子 2 结构在分 子 1 的基础之上引入了既具有强给电子性能又具有刚性 结构的苯胺作为给体的修饰基团. 一方面具有较好平面 性的苯胺作为一个辅助给体能够有利于苯胺中电子更 好地流向给体, 同时苯胺中刚性结构的苯环, 拉开了发
色团分子之间的间距，减少偶极作用，有利于极化时分 子的取向排列, 得到更高的一阶超极化率 $\beta$ 值的同时实 际所测的 $r_{33}$ 值也最高 ${ }^{[15 a]}$.
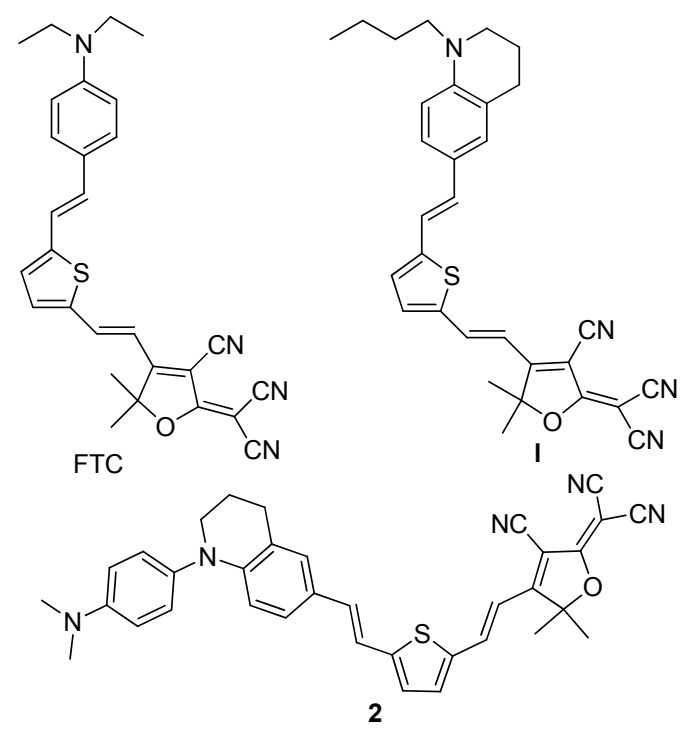

图 4 发色团 FTC, 1 和 2 分子结构式

Figure 4 Chemical structures of FTC, 1 and 2

另外, 本课题组还对 FTC 发色团进行了给体结构 上的其他修饰, 实现了新的双给体结构(图 5). 通过主客体掺杂，将三种发色团 FTC、分子 3 及分子 4 分别掺 杂在无定型的聚碳酸酯聚合物中, 测得宏观电光系数 $r_{33}$ 值分别为 $39,149,143 \mathrm{pm} / \mathrm{V}$, 发色团分子 3 和分子 4 的 $r_{33}$ 值将近是 FTC $r_{33}$ 值的 4 倍. 研究结果表明在发色 团分子 3 和分子 4 的分子结构中, 在苯胺为给体的基础 上新增一个具有刚性结构的苯胺基团，一方面起到
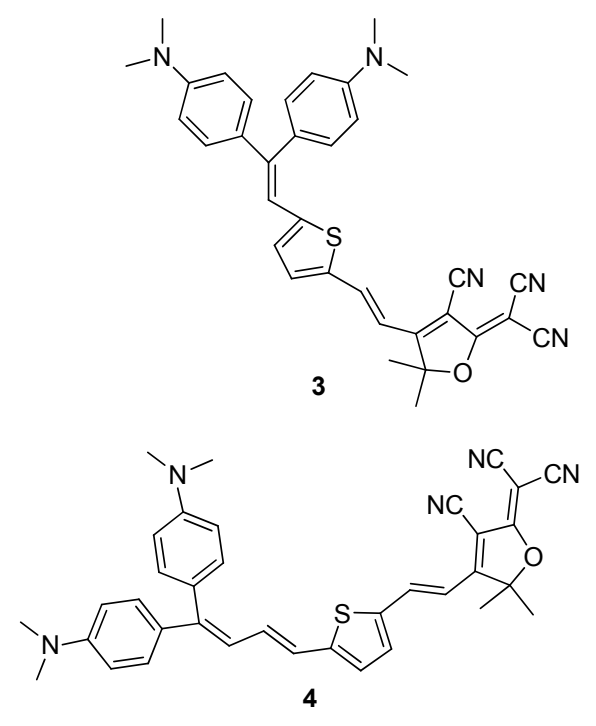

图 5 发色团 3 和 4 分子结构式

Figure 5 Chemical structures of $\mathbf{3}$ and $\mathbf{4}$ 
辅助给体的作用, 可以提高整个分子内的电子云的密 度；同时因为其刚性结构的苯环，具有一定的位阻效应， 起到一个隔离基团的作用, 减少分子之间的偶极一偶极 相互作用, 从而提高极化效率, 获得更高的电光系数 $r_{33}$ 值 $^{[36]}$.

Dalton 等 ${ }^{[33}$ 报道过将发色团真正应用到高分子电 光器件中, 需要满足力学稳定性、热稳定性、化学稳定 性以及光化学稳定性. 高分子材料能否应用于实际器件 中首先需要满足长时间能够承受 $100{ }^{\circ} \mathrm{C}$ 以上的高温. Dalton 课题组 ${ }^{[33]}$ 对以苯胺为给体的发色团做了一系列 的修饰, 在其给体引入不同的柔性支链以及具有刚性结 构的苯环基团作为隔离基团, 并和三苯胺为给体的发色 团进行对比, 合成 8 种发色团(图 6), 其分子结构、分解 温度 $T_{\mathrm{d}}$ 和一阶超极化率如表 1 所示. 结果表明对苯胺为 给体发色团进行修饰, 在 $\mathrm{N}$ 原子中引入一些具有一定位 阻的隔离基团, 会提高分子的分解温度, 其中以三苯胺 为给体的发色团分解温度最高, $T_{\mathrm{d}}$ 可达 $367{ }^{\circ} \mathrm{C}$, 同时测 得三苯胺为给体的发色团也具有较好的一阶超极化率, 因此在高分子电光材料中有较大的应用前景. 同时发色

表 1 发色团分子 $5 \sim 12$ 的性能参数值 ${ }^{[33]}$

Table 1 Performance of the choromophores $5 \sim \mathbf{1 2}^{[33]}$

\begin{tabular}{ccc}
\hline Chormophore & $T_{\mathrm{d}} /{ }^{\circ} \mathrm{C}$ & $\mu \beta_{1.9 \mu \mathrm{m}} /\left(10^{-48} \mathrm{esu}\right)$ \\
\hline $\mathbf{5}$ & 274 & 6200 \\
$\mathbf{6}$ & 296 & 2700 \\
$\mathbf{7}$ & 325 & 2500 \\
$\mathbf{8}$ & 354 & 1300 \\
$\mathbf{9}$ & 268 & 2520 \\
$\mathbf{1 0}$ & 322 & 1211 \\
$\mathbf{1 1}$ & 313 & 1720 \\
$\mathbf{1 2}$ & 367 & 2570 \\
\hline
\end{tabular}

才分子的空间形状会大大影响分子之间的静电作用力, 因此，可以通过改变分子的空间形状达到减少由于分子 之间的相互作用力引起的堆积.

三苯胺为给体的发色团与苯胺为给体的发色团相 比，苯胺中 $\mathrm{N}$ 原子上连接的柔性链被刚性的苯环所取 代，两个苯环的引入一方面起到了隔离基团的作用，提 高了整个分子的空间位置，增大了分子与分子之间的距 离; 同时由于苯环的刚性结构，大大提高了整个分子的 热稳定性能. 因此，后续不断踊跃出以三苯胺作为给体 的发色团的文章.

Chen 等 ${ }^{[37]}$ 以三苯胺为给体, 2-dicyanomethylene-3cyano-4,5-dimethyl-5-trifluoromethyl-2,5-dihydrofuran $\left(\mathrm{CF}_{3}-\mathrm{TCF}\right)$ 为受体，分别选用异佛尔酮以及噻吩为桥, 通过对给体进行修饰, 引入尺寸大小不同的隔离基团 (图 7). 实验测试分析其性能见表 2 , 结果表明通过在给 体引入不同隔离基团能够有效增加一阶微观超极化率 向宏观电光系数转化的效率, 化合物 $\mathbf{2 0}$ 的电光系数 $r_{33}$ 值已达到 $169 \mathrm{pm} / \mathrm{V}$ ，接近于化合物 $17 r_{33}$ 值的 4 倍.

表 2 发色团分子 $13 \sim 20$ 的性能参数值 ${ }^{[37]}$

Table 2 Performance of the choromophores $\mathbf{1 3} \sim \mathbf{2 0}^{[37]}$

\begin{tabular}{ccc}
\hline Chromophore & $T_{\mathrm{d}} /{ }^{\circ} \mathrm{C}$ & $r_{33} /\left(\mathrm{pm} \cdot \mathrm{V}^{-1}\right)$ \\
\hline $\mathbf{1 3}$ & 90 & 19 \\
$\mathbf{1 4}$ & 103 & 27 \\
$\mathbf{1 5}$ & 84 & 37 \\
$\mathbf{1 6}$ & 98 & 60 \\
$\mathbf{1 7}$ & 89 & 47 \\
$\mathbf{1 8}$ & 104 & 60 \\
$\mathbf{1 9}$ & 114 & 106 \\
$\mathbf{2 0}$ & 75 & 169 \\
\hline
\end{tabular}<smiles>CCN(CC)c1ccc(/C=C/c2ccc(C(C#N)=C(C#N)C#N)s2)cc1</smiles>

5<smiles>CN(C)c1ccc(-c2ccc(C(=C(C#N)C#N)c3ccc(-c4ccc(N(C)C)cc4)s3)s2)cc1</smiles><smiles>CCN(CC)c1ccc(-c2ccc(C(C#N)=C(C#N)C#N)s2)cc1</smiles>

6<smiles></smiles>

7<smiles>CCn1c2ccccc2c2cc(/C=C/C3=CC(=C(C#N)C#N)CC(C)(C)C3)ccc21</smiles><smiles>CC1(C)CC(/C=C/c2ccc(N(c3ccccc3)c3ccccc3)cc2)=CC(=C(C#N)C#N)C1</smiles><smiles></smiles>

图 6 发色团 5 12 的分子结构式

Figure 6 Chemical structures of the chromophores $5 \sim 12$ 


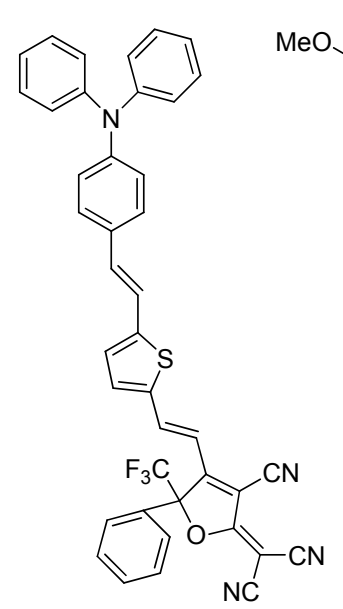

13

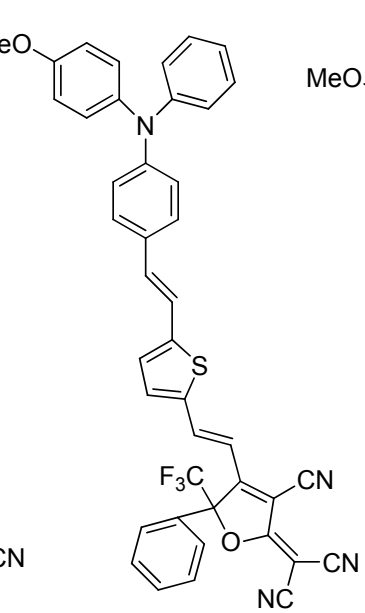

14
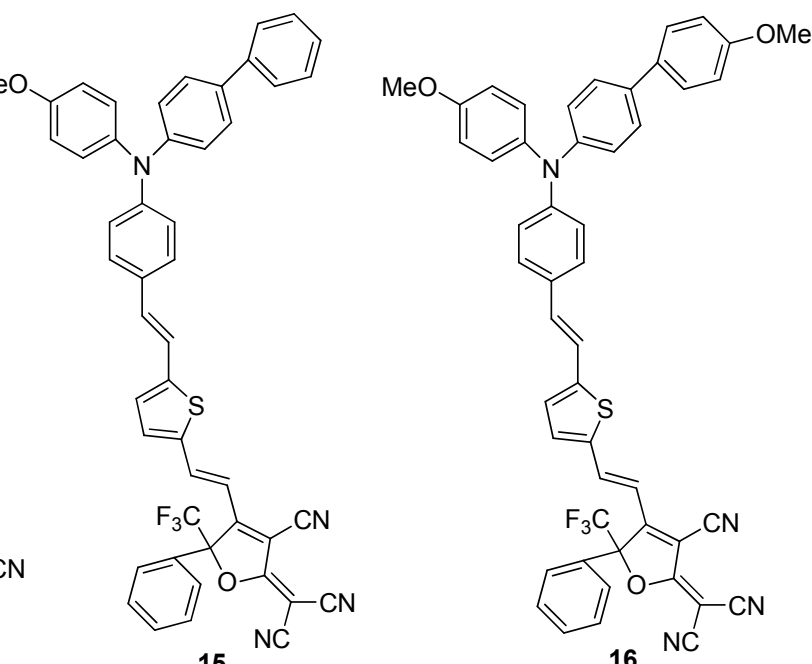

15

16

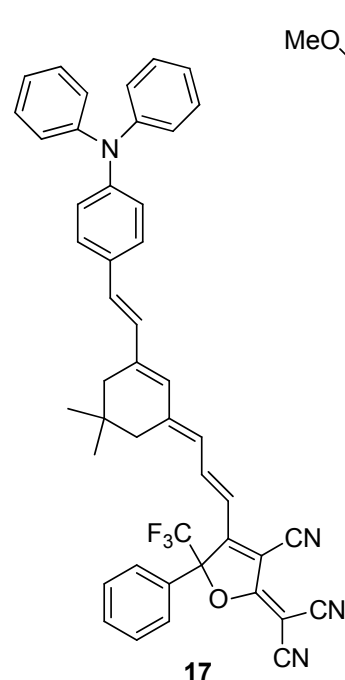

17

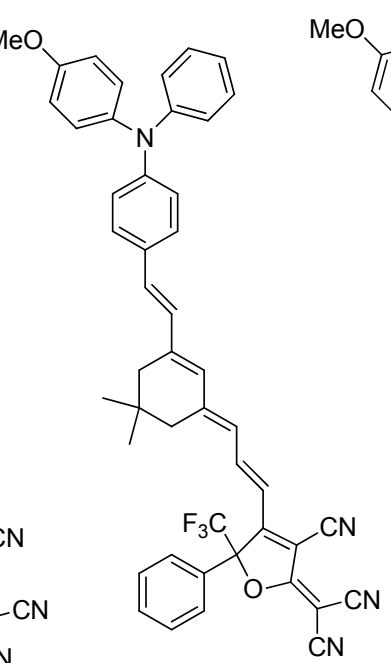

18

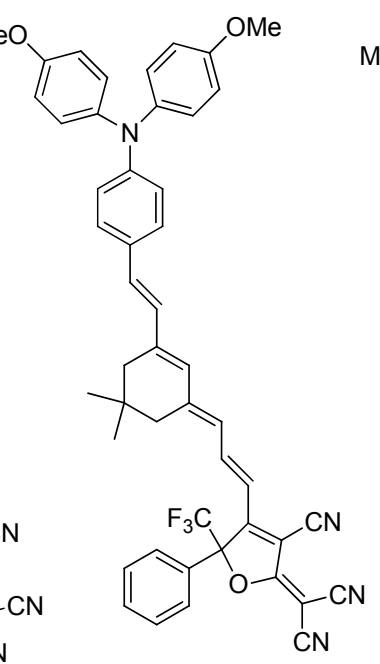

19

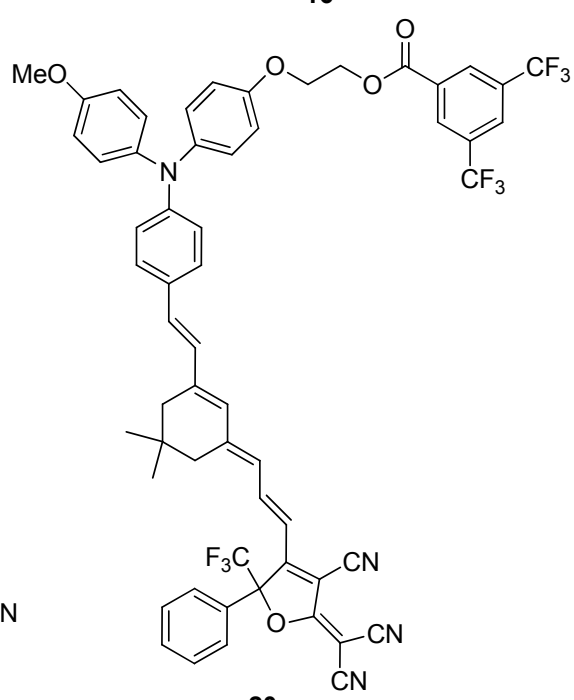

20

图 7 发色团 13 20 的分子结构式

Figure 7 Chemical structures of the chromophores $\mathbf{1 3} \sim \mathbf{2 0}$

本课题组 ${ }^{[15 a]}$ 也对以三苯胺为给体, 噻吩为共轭桥, $\mathrm{TCF}$ 为受体的发色团分子进行修饰, 在三苯胺给体中引 入具有杂原子的支链, 探究隔离基团对发色团性能的影 响(图 8). 发色团分子 21, 22 和 23 都保留了三苯胺作为 给体所拥有的优点即较高的分解温度 $T_{\mathrm{d}}$, 分别为 301 , 310 和 $289{ }^{\circ} \mathrm{C}$. 将三种发色团主客体掺杂到高分子聚合 物中测试其 $r_{33}$ 值分别为 16,58 和 $95 \mathrm{pm} / \mathrm{V}$. 发色团分子 22 和 23 的分子结构相比较 21 的而言, 引入了含有杂原 子的柔性支链. 具有杂原子的柔性支链一方面能够起到 隔离基团的效果, 减少分子之间的偶极相互作用; 另一 方面也起到一个辅助给体的效果, 增加分子内电子的密

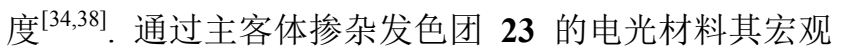
电光系数 $r_{33}$ 值要大于主客体掺杂分子 $\mathbf{2 2}$ 的高分子聚合 物的 $r_{33}$ 值, 这强有力地说明了含有 $\mathrm{N}$ 原子的隔离基团 修饰的给体具有很好的给电子性能, 能够提高发色团的 微观一阶超极化率 $\beta$ 值, 同时又具有很好的隔离效果,
可实现微观一阶超极化率向宏观电光活性的有效转化. 随着研究的深入, 设计并合成给电子能力更强的新型电 子给体成了亟待解决的难题, 为此本课题组 ${ }^{\left[{ }^{[39}\right.}$ 首次合 成了以久洛尼定及其衍生物为新型给体的一系列发色 团分子，从计算的分子一阶超极化率可以看出此种给体 给电子能力要比苯胺高. 将发色团分子 24 和 25 (图 9) 通 过主客体掺杂到高分子聚合物中, 当发色团 24 掺杂浓 度为 $25 \%$ 时, 产生宏观电光系数 $r_{33}$ 值为 $28 \mathrm{pm} / \mathrm{V}$, 当掺 杂浓度为 $40 \%$ 时, 具有最大的宏观电光系数 $r_{33}$ 值, 为 $36 \mathrm{pm} / \mathrm{V}$. 而发色团 25 在掺杂浓度为 $25 \%$ 时, 已到达最 大宏观电光系数 $r_{33}$, 为 $16 \mathrm{pm} / \mathrm{V}$. 以久洛尼定为给体的 发色团产生相对较大的宏观电光系数 $r_{33}$ 值, 是由于久 洛尼定具有较强的给电子能力 ${ }^{[40]}$, 并且具有较好的空 间位阻效应, 大大增加聚合物电光材料中的 $r_{33}$ 值, 因 此, 本课题围绕以久洛尼定为给体的发色团做了大量的 研究工作, 以不断提高材料的性能. 

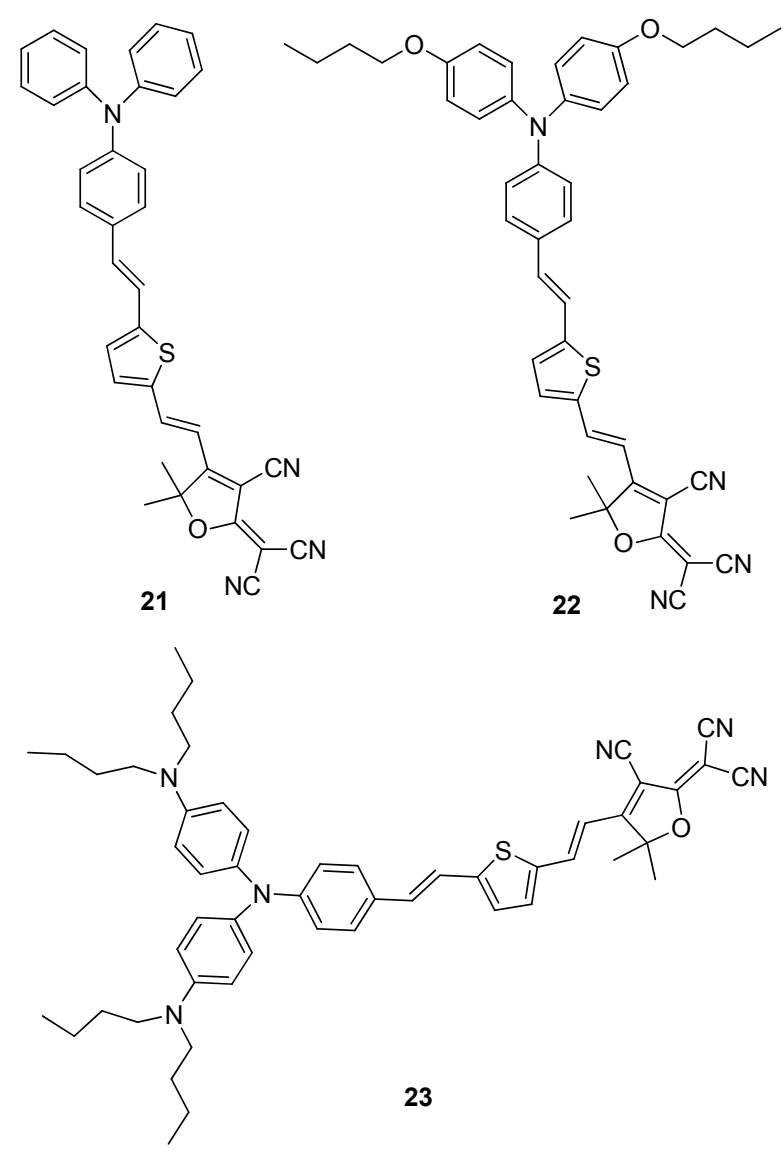

图 8 发色团 $21 \sim 23$ 的分子结构式

Figure 8 Chemical structures of the chromophores $21 \sim 23$

为了进一步提高久洛尼定类发色团产生的宏观电 光性能, 本课题组 ${ }^{[40 b]}$ 对以久洛尼定为给体的发色团进 行修饰，在其给体中引入不同位阻的隔离基团，使发色 团具有不同的位阻效应和分子移动性. 探究改变材料的 极化条件来平衡发色团的位阻效应和分子移动性，从而 获得材料最大的电光系数(图 10). 发色团 27 给体中的酚 羟基被柔性的已醇所取代, 而在发色团 $\mathbf{2 6}$ 中, 酚羟基除 了被柔性的己醇所取代外，末端的羟基被大位阻基团叔 丁基-二甲基氯硅烷(TBDMSCl)所取代形成硅烷封端.
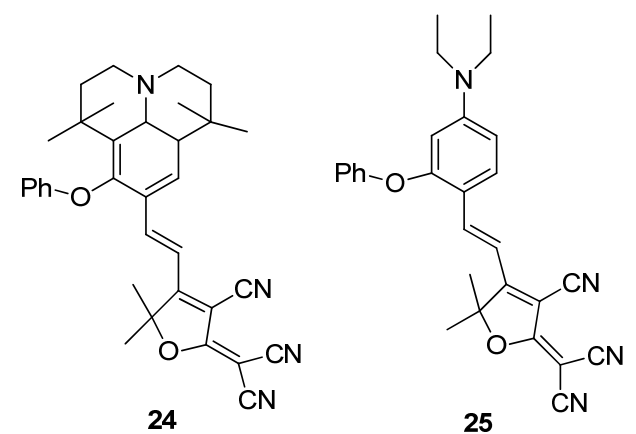

图 9 发色团 24 和 25 的分子结构式

Figure 9 Chemical structures of the chromophores 24 and 25

发色团 28 是用常见的刚性基团芐基修饰. 将这三种发 色团通过主客体掺杂到聚碳酸酯 APC-1 和 APC-2 中，分 别对电光系数进行测试, 结果见表 3. 发色团 26 在聚碳 酸酯 APC-1 和 APC-2 中都具有最高的电光系数 $r_{33}$ 值. 发色团 27 中拥有最强位阻效应，确并没有产生最高的 电光系数, 说明并不是含有最强位阻效应的发色团的电 光材料最具优势. 发色团 27 中己醇支链末端羟基被大 硅烷取代，使得整个隔离基团链又长体积又大，容易造 成发色团与周围的聚合物链缠绕在一起, 反而减弱了发 色团分子的移动性，导致发色团在电场诱导下不能有效 地有序排列. 因此利用隔离基团对发色团结构进行修 饰, 需要平衡位阻和分子移动性之间的关系, 使发色团 在材料中展示出较高的宏观电光系数.

\section{2 隔离基团修饰发色团共轭桥}

Kim 等 ${ }^{[41]}$ 利用 “Diels-Alder” click 反应，活化发色 团共轭桥中间位置，改变了发色团与高分子的连接位 点，扩大了隔离基团的修饰范围，能够灵活改变发色团 的形状，制造了一系列大分子，与高聚物主链成键. 此 外, Kim 等总结了将发色团在共轭桥位点处与主链成键 所形成的高分子情况(图 11), 为后续的研究者们合成枝 状型聚合物提供有利的参考.
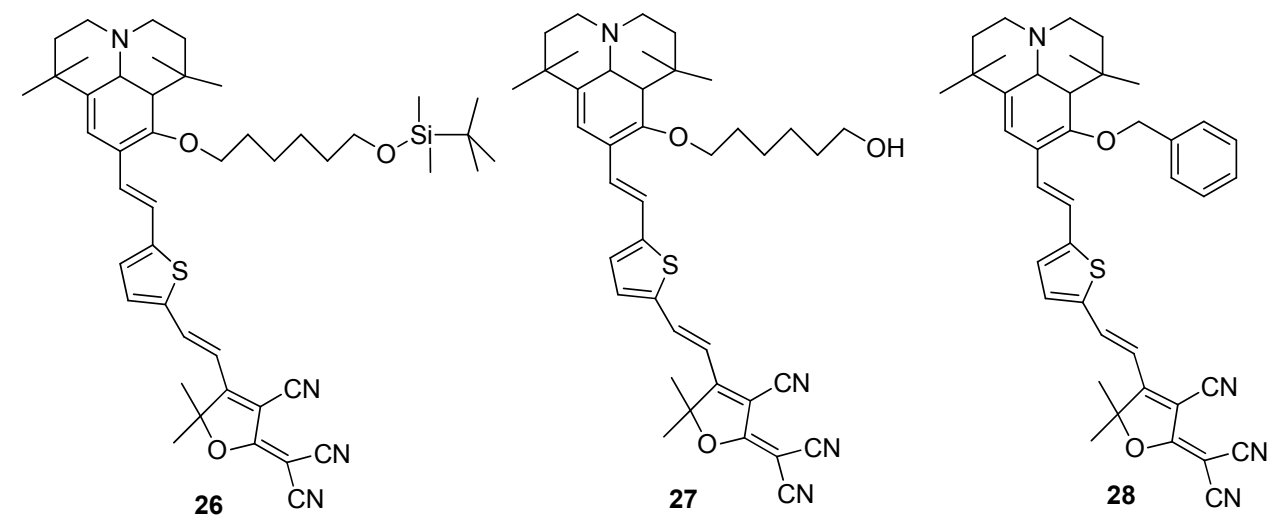

图 10 发色团 26 28 的分子结构式

Figure 10 Chemical structures of the chromophores $\mathbf{2 6} \sim \mathbf{2 8}$ 
表 3 材料的性能参数 ${ }^{[40 b]}$

Table 3 The performance of the material ${ }^{[40 \mathrm{~b}]}$

\begin{tabular}{ccc}
\hline Film & $T_{\mathrm{g}}{ }^{\circ} \mathrm{C}$ & $r_{33} /\left(\mathrm{pm} \cdot \mathrm{V}^{-1}\right)$ \\
\hline $\mathbf{2 6} / \mathrm{APC}-1$ & 112 & 89 \\
$\mathbf{2 7} / \mathrm{APC}-1$ & 106 & 114 \\
$\mathbf{2 8} / \mathrm{APC}-1$ & 110 & 47 \\
$\mathbf{2 6} / \mathrm{APC}-2$ & 137 & 266 \\
$\mathbf{2 7} / \mathrm{APC}-2$ & 129 & 127 \\
$\mathbf{2 8} / \mathrm{APC}-2$ & 137 & 53 \\
\hline
\end{tabular}

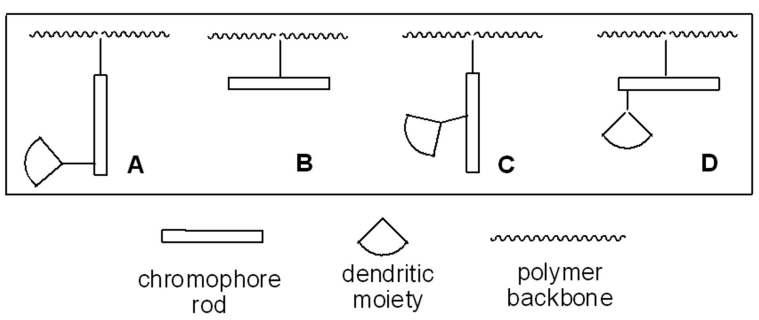

图 11 不同类型枝状高分子的结构示意图 ${ }^{[41]}$

Figure 11 Graphical illustration of various dendronized polymers $^{[41]}$

Cheng 等 ${ }^{[37]}$ 采用苯胺为给体, 异佛尔酮为共轭电子 桥, 同时将受体改为吸电子能力较强的受体 $\mathrm{CF}_{3}-\mathrm{TCF}$. 为了进一步提高发色团的性能, 对异佛尔酮桥进行修 饰, 引入含有杂原子的长支链作为隔离基团, 合成发色 才 29(图 12). 利用主客体掺杂的方式, 测得发色团分子 29 的 $r_{33}$ 值为 $219 \mathrm{pm} / \mathrm{V}$. 在异佛尔酮桥中引入具有硫原 子的长支链作为隔离基团, 使得发色团分子均具有全反 式的双键, 有利于增强共轭效应, 利于电子的传输 ${ }^{[42]}$.

在此基础之上, 为了进一步研究隔离基团对分子形 状的影响, 改善发色团性能. 本课题组 ${ }^{[38]}$ 也曾对发色团 的共轭桥上引入支链隔离基团进行研究. 我们在噻吩桥 上引入支链, 一方面能够改善发色团分子的形状, 有效 地抑制分子在极化过程中产生的静电作用, 减少分子的
堆积; 另一方面柔性支链的引入有利于提高了发色团分 子的溶解度. 发色团分子 30 32 (图 13) 通过主客体掺 杂在高分子聚合物中，测得的 $r_{33}$ 值分别为 149,138 和 $157 \mathrm{pm} / \mathrm{V}$. 实验结果验证了在共轭桥处引入柔性长支链 增加了发色团的溶解性能, 但是发色团分子 31 相比较 发色团分子 30 而言, 材料的电光系数 $r_{33}$ 值反而下降, 说明了并非引入隔离基团都能够很好地提高发色团电 光活性，对于不同的发色团应该选择适合的隔离基团进 行修饰. 此外, 通过对比分析发色团 33 和 34 的性能, 研 究隔离基团所处的位置对于材料电光活性的影响. 将发 色团分子 33 和 34 通过主客体掺杂在高分子电光材料中, 测得 $r_{33}$ 值为 $163,106 \mathrm{pm} / \mathrm{V}$. 发色团分子 33 通过主客体 掺杂的聚合物有较大的 $r_{33}$ 值, 是因为含杂原子的柔性 链靠近给体，除了起到隔离基团的作用，同时还具有一 定的给电子性能, 能够增大整个发色团分子中的电子密 度, 从而使得宏观电光材料的 $r_{33}$ 值较大. 而发色团分子 34 通过主客体掺杂的聚合物的 $r_{33}$ 值明显降低可能是由 于支链中的杂原子靠近受体，具有吸电子性，减少了发 色团分子内的电子密度．该工作在 “suitable isolation group” 理论基础上探究了隔离基团的位置对发色团性 能的改善的影响, 为后续进一步研究隔离基团修饰发色 团分子提供理论依据.

Hammon 等 ${ }^{[32]}$ 采用苯胺为给体, $\mathrm{TCF}$ 为受体, 双噻 吩为共轭桥, 并且对双噻吩进行修饰, 引入隔离基团. 实验分析表明这 3 种发色团(图 14)具有较好的溶解性, 测得发色团分子 35,36 和 37 的 $r_{33}$ 值分别为(52 \pm 10$)$, $(31 \pm 6)$ 和 $(17 \pm 3) \mathrm{pm} / \mathrm{V}$. 相比较发色团分子 37 , 发色团 分子 35 和 36 的 $r_{33}$ 值都有所增加. 说明对发色团分子的 结构进行修饰，引入具有一定位阻效应的隔离基团，能 够大大增强发色团在材料中的电光活性. 另外发色团 35 和 36 产生的不同电光系数也说明了不同的隔离基团

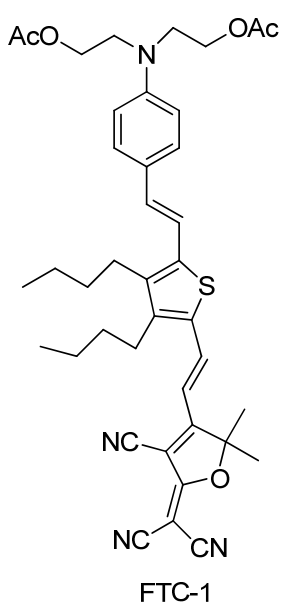

FTC-1

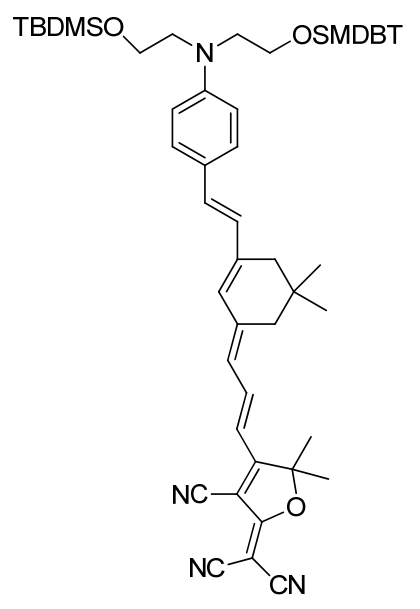

CLD-1 trans isomer

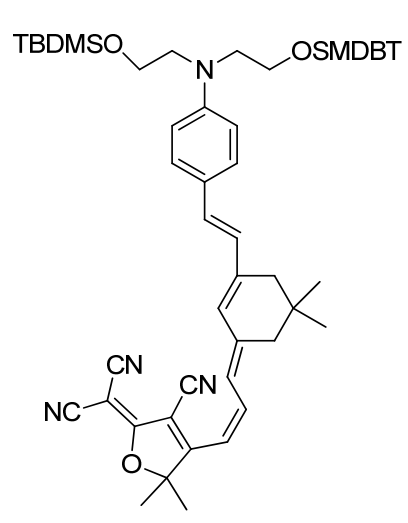

CLD-1 cis isomer

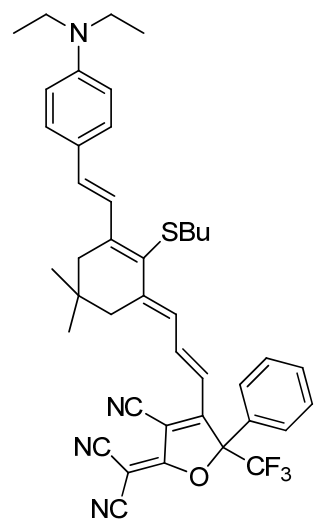

29

图 12 发色团 FTC-1, CLD-1 和 29 的分子结构式

Figure 12 Chemical structures of the chromophores FTC, CLD-1 and 29 
<smiles>CCN(CC)c1ccc(C(=Cc2ccc(C=CC3=C(C#N)C(=C(C#N)C#N)OC3(C)C)s2)c2ccc(N(CC)CC)cc2)cc1</smiles><smiles>CCN(CC)c1ccc(C(=Cc2sc(C=CC3=C(C#N)C(=C(C#N)C#N)OC3(C)C)cc2OCCO[Si](C)(C)C(C)(C)C)c2ccc(N(CC)CC)cc2)cc1</smiles>

33<smiles>CCCCCCc1cc(/C=C/C2=C(C#N)C(=C(C#N)C#N)OC2(C)C)sc1C=C(c1ccc(N(CC)CC)cc1)c1ccc(N(CC)CC)cc1</smiles>

31<smiles>CCN(CC)c1ccc(C(=Cc2cc(OCCO[Si](C)(C)C(C)(C)C)c(C=CC3=C(C#N)C(=C(C#N)C#N)OC3(C)C)s2)c2ccc(N(CC)CC)cc2)cc1</smiles><smiles>CCCCCCOc1cc(/C=C/C2=C(C#N)C(=C(C#N)C#N)OC2(C)C)sc1C=C(c1ccc(N(CC)CC)cc1)c1ccc(N(CC)CC)cc1</smiles>

32

图 13 发色团 30 34 的分子结构式

Figure 13 Chemical structures of the chromophores 30 $\sim 34$

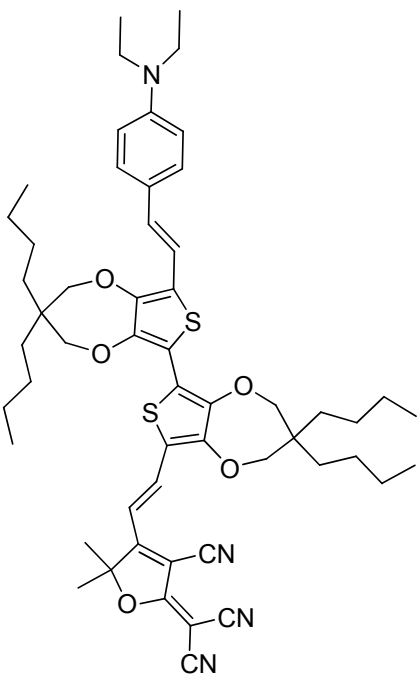

35

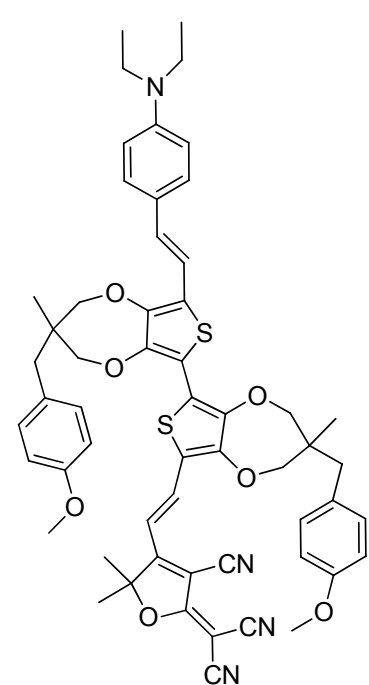

36

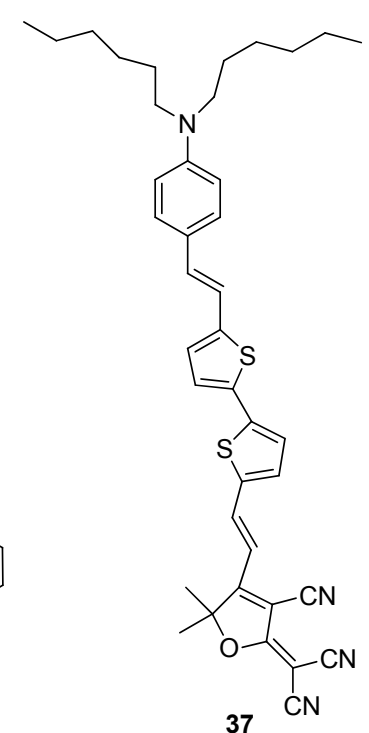

37

图 14 发色团 35 37 的分子结构式

Figure 14 Chemical structures of the chromophores $35 \sim 37$

修饰发色团, 效果不同, 因此选择最佳合适基团能够最 大化地提高发色团的电光活性.

尽管对发色团分子结构进行修饰, 引入隔离基团是 目前最热门的研究方法, 然而一些柔性基团对于增大分 子之间的距离, 减少分子在极化过程中的堆积的效果不 是很理想, 导致微观一阶超极化率 $\beta$ 值向宏观电光系数 $r_{33}$ 转换的效率不高 ${ }^{[39]}$. 本课题组 ${ }^{[23]}$ 采用具有刚性结构 且连有杂原子基团的苯环为隔离基团在桥上修饰以久 洛尼定为给体 TCF 为受体的发色团, 旨在提高发色团 宏观光电性能、稳定性以及溶解性. 通过热重实验分析 测试这三种发色团分子 $38 \sim 40$ (图 15) 都具有较好的热 稳定性能, $T_{\mathrm{d}}$ 分别为 241,273 和 $265{ }^{\circ} \mathrm{C}$. 将这三种发色 
团通过主客体掺杂在高聚物中, 相比于没有使用刚性隔 离基团的发色团 38(电光系数为 $121 \mathrm{pm} / \mathrm{V}$ ), 采用刚性隔 离基团修饰的发色团 39 和 40 的宏观电光系数分别为 $197,201 \mathrm{pm} / \mathrm{V}$. 表明在双键桥上引入刚性苯环结构作为 隔离基团，能够高效地抑制分子之间的相互作用，从而 利于提高发色团的宏观电光系数, 同时还可以增加分子 的稳定性能, 提高 $T_{\mathrm{d}}$ 值, 此种材料在器件制作方面具有 十分广泛的应用前景.

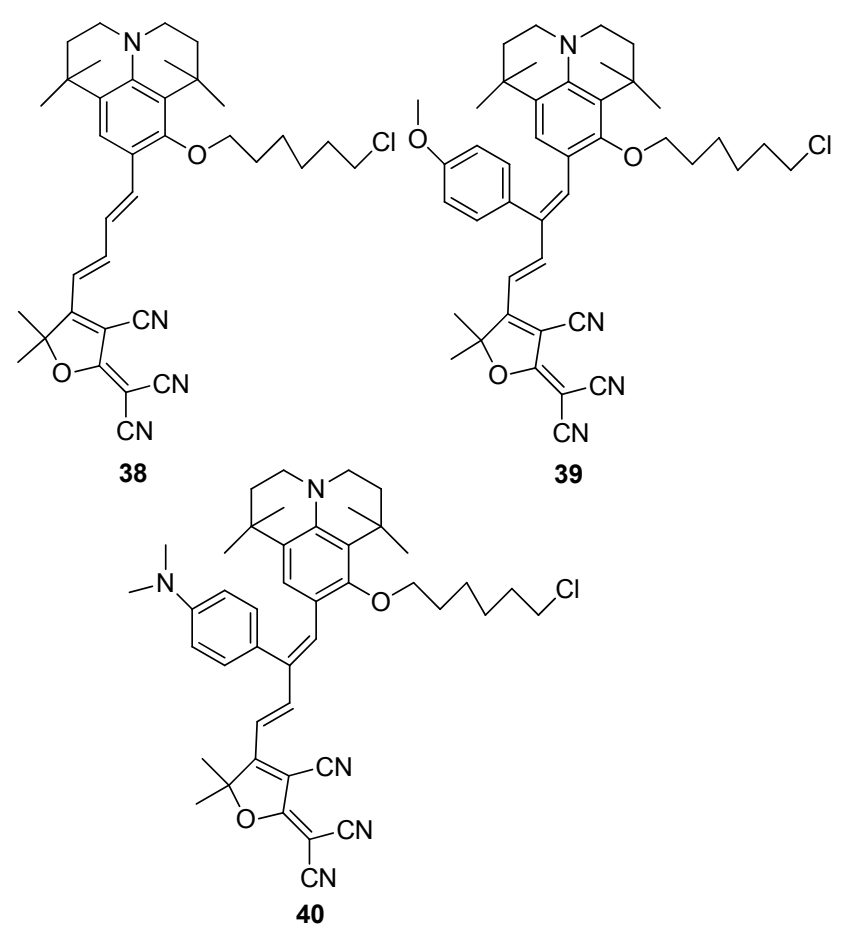

图 15 发色团 $38 \sim 40$ 的分子结构式

Figure 15 Chemical structures of the chromophores $38 \sim \mathbf{4 0}$

另外, 有些工作是利用聚合物结构作为隔离基团,
比如为了更好地发挥久洛尼定为给体的优势, 本课题 组 ${ }^{[43]}$ 对以久洛尼定为给体, TCF 为受体的发色团进行修 饰，加入了具有枝状形的刚性隔离基团，此外在隔离基 才的末端连接具有 $\mathrm{OH}$ 的柔性链(图 16). 聚合物组成的 枝状形隔离基团一方面具有较大的位阻可以增加分子 之间的距离, 减少分子堆积; 另一方面发色团与聚合物 成键, 避免了主客体掺杂过程中可能存在的相分离. 当 发色团与高分子聚合物以 $1: 2.4$ 成键时, 测得 $r_{33}$ 值达 到 $55 \mathrm{pm} / \mathrm{V}$.

武汉大学李振课题组 ${ }^{[44]}$ 研究了具有不同尺寸的隔 离基团对于 NLO 性能的影响. 选择两种具有相同给体 以及共轭桥的发色团分子, 发色团分子苯胺给体中的两 个 $\mathrm{OH}$ 与聚合物单体成键，嵌入在高分子聚合物中. 通 过改变与电子桥相连的隔离基团的尺寸大小, 总共合成 了 10 种高分子聚合物(图 17). 对比了不同的隔离基团对 以硝基和硫酰基为受体的两种系列聚合物的 $d_{33}$ 值的影 响(图 18) ${ }^{[45]}$. 从图中可以直观得出发色团分子的隔离基 才会直接影响着最后宏观电光材料 $d_{33}$ 值的大小. 由于 硫酰基受体弱于硝基受体，因此硝基为受体的发色团分 子之间的作用力更强. 在相同的极化条件下，硝基为受 体的发色团分子的定向排列更难进行. 因此，对于硝基 为受体的发色团而言，当隔离基团的尺寸增大为咔唑 时，其聚合物才具有最大的 $d_{33}$ 值，随着隔离基团的尺 寸增大, 发色团在聚合物中的浓度较低, 因此 $d_{33}$ 值又 下降. 而对于以硫酰基为受体的发色团而言，由于其分 子间的作用力没有硝基发色团分子之间的作用力大，故 当隔离基团为苯基时，已经达到最好的效果，即具有最 大的 $d_{33}$ 值, 随着隔离基团的增大, 发色团在聚合物中 的浓度降低, 因此其聚合物的宏观电光系数 $d_{33}$ 值开始 下降. 该研究工作很好地说明了特定的发色团具有最适

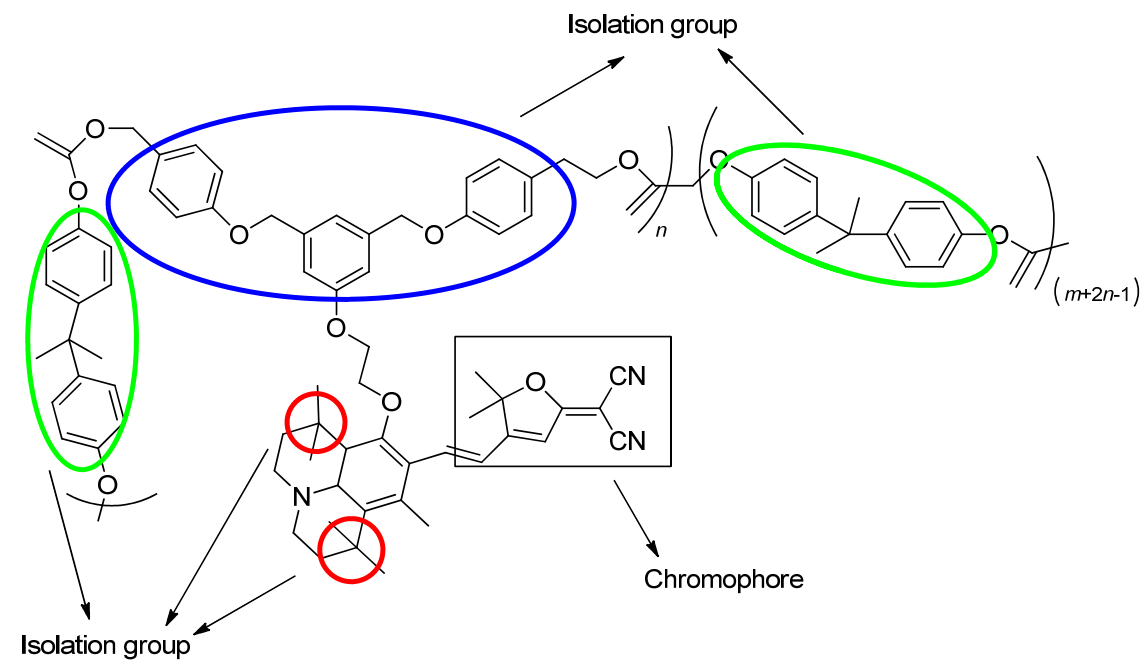

图 16 发色团 41 分子结构图 ${ }^{[43]}$

Figure 16 Chemical structure of the chromophore $41^{[43]}$ 


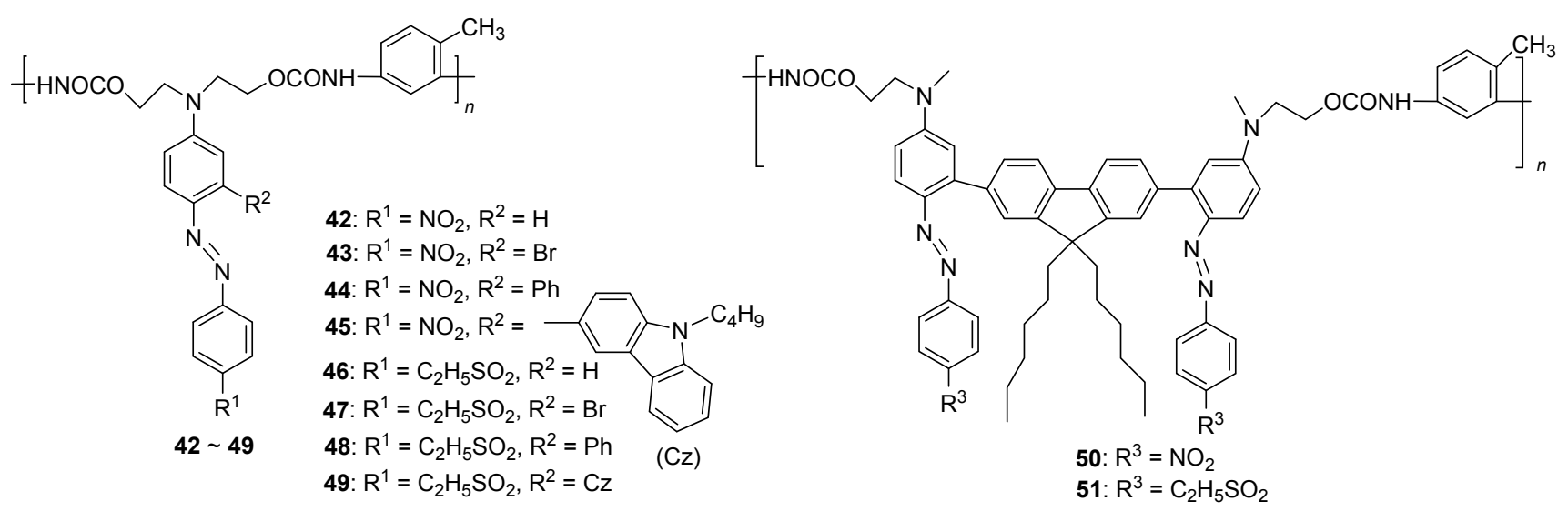

图 17 枝状高分子 $\mathbf{4 2 \sim 5 1}$ 的分子结构图

Figure 17 Chemical structure of dendronized polymers $\mathbf{4 2} \sim \mathbf{5 1}$

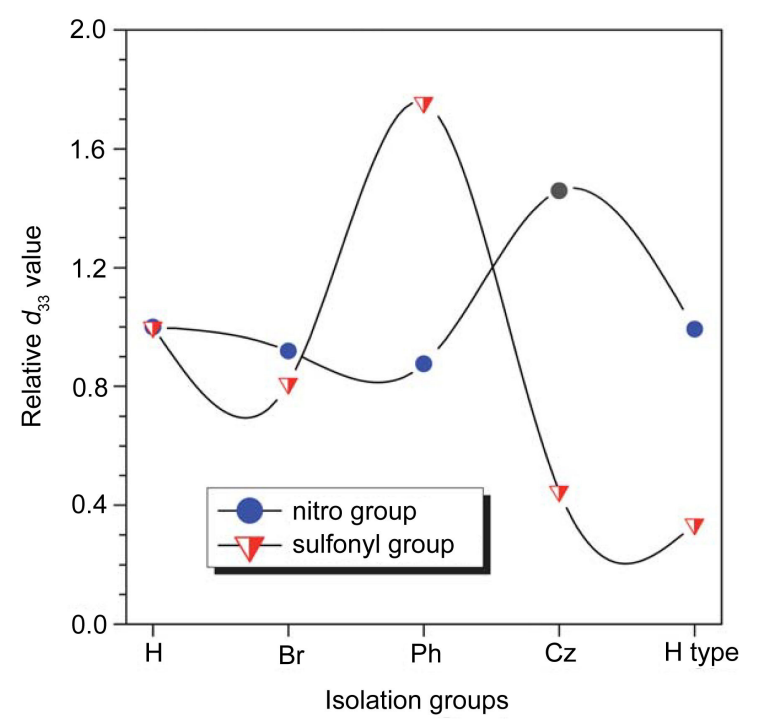

图 18 含不同尺寸大小间隔基团高分子 $\mathbf{4 2} \sim \mathbf{5 1}$ 的 $d_{33}$ 值比较 Figure 18 Comparison of the $d_{33}$ value of dendronized polymers $\mathbf{4 2} \sim \mathbf{5 1}$ containing different size isolation spacers

隔离基团, 即 “suitable isolation group” [45], 为后续研究 有机非线性光学材料的研究奠定了理论基础.

\section{3 总结与展望}

迄今为止, 如何将微观一阶超极化率 $\beta$ 值高效地转 化为宏观电光系数 $r_{33}$ 值, 一直是专家学者们研究的主 要问题. 对近十几年关于隔离基团修饰有机非线性光学 发色团的研究进行较为全面的总结. 隔离基团的引入一 方面能够增加发色团之间的距离, 有效减少分子之间因 静电相互作用产生的堆积, 提高材料的电光性能; 另一 方面通过对隔离基团进行一些修饰，包括在隔离基团中 增加一些柔性支链, 增加杂原子或者官能团 $\mathrm{OH}$ 等, 来 提高发色团分子其他化学性能如溶解性、热稳定性、透 光性等; 甚至还有一些专家学者们通过隔离基团将 2 个
乃至更多的发色团分子连接起来, 形成 $\mathrm{X}$ 型、 $\mathrm{H}$ 型以及 星型等形状的大分子发色团，丰富了发色团分子种类. 可是, 要实现市场的商品化, 仍需要解决以下几点问题: (1)在提高发色团分子一阶超极化率的同时会伴随着光 损耗的增大，故提高非线性的同时减少光损耗成为设计 与合成发色团中的一个关键问题; (2)在发色团分子中引 入隔离基团可能影响发色团分子在高分子聚合物中的 浓度，从而导致一阶微观超极化率向宏观光电系数 $r_{33}$ 转化的实际效率不高, 因此, 在合成分子的时候需要寻 找合适的隔离基团，提高 $\beta$ 向 $r_{33}$ 的实际转化效率. 通过 研究者们的努力, 有望合成出综合性能优异的发色团, 进而开展其应用研究.

\section{References}

[1] Zhang, A.-R.; Xiao, H.-Y.; Cong, S.-Y.; Zhang, M.-L.; Zhang, H.; Bo, S.-H.; Wang, Q.; Zhen, Z.; Liu, X.-H. J. Mater. Chem. C 2015, 3, 370 .

[2] Chen, D.-T.; Fetterman, H. R.; Chen, A.-T.; Steier, W. H.; Dalton, L. R.; Wang, W.-S.; Shi, Y.-Q. Appl. Phys. Lett. 1997, 70, 3335.

[3] Ding, R.; Baehr-Jones, T.; Kim, W.-J.; Spott, A.; Fournier, M.; Fedeli, J.-M.; Huang, S.; Luo, J.-D.; Jen, A. K.-Y.; Dalton, L.; Hochberg, M. J. Lightwave Technol. 2011, 29, 1112.

[4] Liang, P.-X.; Mi, Y.-S.; Duan, J.-S.; Yang, Z.; Wang, D.; Cao, H.; He, W.-L. Chin. J. Chem. 2016, 34, 381.

[5] (a) Sullivan, P.-A.; Dalton, L.-R. Acc. Chem. Res. 2010, 43, 10. (b) Chen, L.; Tang, X.; Jia, K.; Tang, X.-Z. Chin. J. Org. Chem. 2016, 36, 2197 (in Chinese). (陈垒, 唐翔, 贾坤, 唐先忠, 有机化学, 2016, 36, 2197.)

[6] Li, Q.-Q.; Qin, J.-G.; Li, Z. Polym. Bull. 2010, 3 (in Chinese). (李倩倩，秦金贵，李振，高分子通报, 2010, 3.)

[7] Chou, M.-J.; Choi, D.-H.; Sullivan, P. A.; Akelaitis, A. J. P.; Dalton, L. R. Prog. Polym. Sci. 2008, 33, 1013.

[8] (a) Bai, Y.-W.; Song, N.-H.; Gao, J.-P.; Sun, X.; Wang, X.-M.; Yu, G.-M.; Wang, Z.-Y. J. Am. Chem. Soc. 2005, 127, 2060.

(b) Dalton, L. In Polymers for Photonics Applications I, Ed.: Lee, K.-S., Springer Berlin Heidelberg, Berlin, Heidelberg, 2002, p. 1.

[9] (a) Shi, Y.-Q.; Zhang, C.; Zhang, H.; Bechtel, J. H.; Dalton, L. R.; Robinson, B. H.; Steier, W. H. Science 2000, 288, 119.

(b) Sinyukov, A. M.; Leahy, M. R.; Hayden, L. M.; Haller, M.; Luo, J.-D.; Jen, A. K.-Y.; Dalton, L. R. Appl. Phys. Lett. 2004, 85, 5827.

[10] Pereverzev, Y. V.; Gunnerson, K. N.; Prezhdo, O. V.; Sullivan, P. A.; 
Liao, Y.; Olbricht, B. C.; Akelaitis, A. J. P.; Jen, A. K. Y.; Dalton, L. R. J. Phys. Chem. C 2008, 112, 4355.

[11] Udupa, A. H.; Erlig, H.; Tsap, B.; Chang, Y.; Chang, D.; Fetterman, H. R.; Zhang, H.; Sang-Shin, L.; Wang, F.; Steier, W. H.; Dalton, L. R. Electron. Lett. 1999, 35, 1702.

[12] Baehr-Jones, T.; Penkov, B.; Huang, J.; Sullivan, P.; Davies, J.; Takayesu, J.; Luo, J.-D.; Kim, T.-D.; Dalton, L.; Jen, A.; Hochberg, M.; Scherer, A. Appl. Phys. Lett. 2008, 92, 163303.

[13] Tian, Y.-Q.; Chen, C.-Y.; Haller, M. A.; Tucker, N. M.; Ka, J.-W.; Luo, J.-D.; Huang, S.; Jen, A. K. Y. Macromolecules 2007, 40, 97.

[14] Baehr-Jones, T.; Hochberg, M.; Wang, G.-X.; Lawson, R.; Liao, Y.; Sullivan, P. A.; Dalton, L.; Jen, A. K. Y.; Scherer, A. Opt. Express $\mathbf{2 0 0 5}, 13,5216$.

[15] (a) Yang, Y.-H.; Liu, F.-G.; Wang, H.-R.; Bo, S.-H.; Liu, J.-L.; Qiu, L.; Zhen, Z.; Liu, X.-H. J. Mater. Chem. C 2015, 3, 5297. (b) Steier, W. H.; Chen,A.-T.; Lee, S.-S.; Garner, S.; Zhang, H.; Chuyanov, V.; Dalton, L. R.; Wang, F.; Ren, A. S.; Zhang, C.; Todorova, G.; Harper, A.; Fetterman, H. R.; Chen, D.; Udupa, A.; Bhattacharya, D.; Tsap, B. Chem. Phys. 1999, 245, 487.

(c) Mina, A. M.; Baez, H.; Martins, G. S. P.; Alayo, M. I. J. Non-Crst. Solids. 2008, 354, 2565;

[16] Clays, K.; Persoons, A. Phys. Rev. Lett. 1991, 66, 2980.

[17] El-Shishtawy, R. M.; Borbone, F.; Al-amshany, Z. M.; Tuzi, A.; Barsella, A.; Asiri, A. M.; Roviello, A. Dyes Pigm. 2013, 96, 45.

[18] (a) Zhang, C.; Dalton, L. R.; Oh, M.-C.; Zhang, H.; Steier, W. H. Chem. Mater. 2001, 13, 3043.

(b) Harper, A. W.; Sun, S.; Dalton, L. R.; Garner, S. M.; Chen, A.; Kalluri, S.; Steier, W. H.; Robinson, B. H. J. Opt. Soc. Am. B: Opt. Phys. 1998, 15, 329.

(c) Dalton, L. R.; Harper, A. W.; Robinson, B. H. Proc. Natl Acad. Sci. U.S. A. 1997, 94, 4842 .

[19] (a) Li, Z.-A.; Li, Z.; Qin, J.-G. Chin. J. Org. Chem. 2008, 28, 975 (in Chinese).

(李忠安，李振，秦金贵，有机化学, 2008, 28,975.)

(b) Koeckelberghs, G.; Sioncke, S.; Verbiest, T.; Severen, I. V.; Picard, I.; Persoons, A.; Samyn, C. Macromolecules 2003, 36, 9736.

[20] Zhang, W.; He, C.-Y.; Xiao, X.-W.; Song, W.-N.; Gao, Y.-C.; Chen, Z.-M.; Dong, Y.-L.; Wu, Y.-Q.; Wang, Q. Chin. J. Chem. 2016, 34, 1006 .

[21] (a) Ma, H.; Liu, S.; Luo, J.-D.; Suresh, S.; Liu, L.; Kang, S. H.; Haller, M.; Sassa, T.; Dalton, L. R.; Jen, A. K.-Y. Adv. Funct. Mater. 2002, 12,565

(b) Wang, H.-R.; Liu, F.-G.; Yang, Y.-H.; Xu, H.-J.; Peng, C.-C.; Bo, S.-H.; Zhen, Z.; Liu, X.-H.; Qiu, L. Dyes Pigm. 2015, 112, 42.

[22] Wu, W.-B.; Fu, Y.-J.; Wang, C.; Xu, Z.; Ye, C.; Qin, J.-G.; Li, Z. Chin. J. Polym. Sci. 2013, 31, 1415.

[23] Hu, C.-L.; Liu, F.-G.; Zhang, H.; Huo, F.-Y.; Yang, Y.-H.; Wang, H.-R.; Xiao, H.-Y.; Chen, Z.; Liu, J.-L.; Qiu, L.; Zhen, Z.; Liu, X.-H.; Bo, S.-H. J. Mater. Chem. C 2015, 3, 11595.

[24] Sullivan, P. A.; Rommel, H.; Liao, Y.; Olbricht, B. C.; Akelaitis, A. J. P.; Firestone, K. A.; Kang, J.-W.; Luo, J.-D.; Davies, J. A.; Choi, D. H.; Eichinger, B. E.; Reid, P. J.; Chen, A.-T.; Jen, A. K. Y.; Robinson, B. H.; Dalton, L. R. J. Am. Chem. Soc. 2007, 129, 7523.

[25] Peng, C.-C.; Wu, J.-Y.; Liu, J.-L.; Qiu, L.; Liu, X.-H.; Bo, S.-H.; Zhen, Z. Polym. Chem. 2013, 4, 2703.

[26] Huang, H.-Y.; Deng, G.-W.; Liu, J.-L.; Wu, J.-Y.; Si, P.; Xu, H.-J.; Bo, S.-H.; Qiu, L.; Zhen, Z.; Liu, X.-H. Dyes Pigm. 2013, 99, 753.
[27] Xu, H.-J.; Zhang, M.-L.; Zhang, A.-R.; Deng, G.-W.; Si, P.; Huang, H.-Y.; Peng, C.-C.; Fu, M.-K.; Liu, J.-L.; Qiu, L.; Zhen, Z.; Bo, S.-H.; Liu, X.-H. Dyes Pigm. 2014, 102, 142.

[28] Sullivan, P. A.; Akelaitis, A. J. P.; Lee, S. K.; McGrew, G.; Lee, S. K.; Choi, D. H.; Dalton, L. R. Chem. Mater. 2006, 18, 344.

[29] (a) Li, Z.-A.; Li, P.-C.; Dong, S.-C.; Zhu, Z.-C.; Li, Q.-Q.; Zeng, Q.; Li, Z.; Ye, C.; Qin, J.-G. Polymer 2007, 48, 3650.

(b) Li, Q.-Q.; Li, Z.; Zeng, F.-X.; Gong, W.; Li, Z.-A.; Zhu, Z.-C.; Zeng, Q.; Yu, S.-S.; Ye, C.; Qin, J.-G. J. Phys. Chem. B 2007, 111, 508.

[30] (a) Wu, W.-B; Qin, J.-G.; Li, Z. Polymer 2013, 54, 4351

(b) Li, Z.; Dong, S.; Yu, G.; Li, Z.; Liu, Y.; Ye, C.; Qin, J. Polymer 2007, 48, 5520 .

(c) Kityk, I. V.; Alzayed, N.; Reshak, A. H.; Plucinski, K. J.; Berdowski, J.; Fuks-Janczarek, I.; Miedzinski, R.; Tylczynski, Z. Funct. Mater. Lett. 2011, 04, 357.

[31] Li, Q.-Q.; Qin, J.-G.; Li, Z. Chin. J. Org. Chem. 2011, 31, 1337 (in Chinese).

(李倩倩, 秦金贵, 李振, 有机化学, 2011, 31, 1337.)

[32] Hammond, S. R.; Clot, O.; Firestone, K. A.; Bale, D. H.; Lao, D.; Haller, M.; Phelan, G. D.; Carlson, B.; Jen, A. K. Y.; Reid, P. J.; Dalton, L. R. Chem. Mater. 2008, 20, 3425.

[33] Robinson, B. H.; Dalton, L. R.; Harper, A. W.; Ren, A.; Wang, F.; Zhang, C.; Todorova, G.; Lee, M.; Aniszfeld, R.; Garner, S.; Chen, A.; Steier, W. H.; Houbrecht, S.; Persoons, A.; Ledoux, I.; Zyss, J.; Jen, A. K. Y. Chem. Phys. 1999, 245, 35.

[34] Yang, Y.-H.; Wang, H.-R.; Liu, F.-G.; Yang, D.; Bo, S.-H.; Qiu, L.; Zhen, Z.; Liu, X.-H. Phys. Chem. Chem. Phys. 2015, 17, 5776.

[35] Liu, F.-G.; Zhang, M.-L.; Xiao, H.-Y.; Yang, Y.-H.; Wang, H.-R.; Liu, J.-L.; Bo, S.-H.; Zhen, Z.; Liu, X.-H.; Qiu, L. J. Mater. Chem. C 2015, 3, 9283.

[36] Luo, J.-D.; Haller, M.; Ma, H.; Liu, S.; Kim, T.-D.; Tian, Y.-Q.; Chen, B.-Q.; Jang, S.-H.; Dalton, L. R.; Jen, A. K. Y. J. Phys. Chem. B 2004, 108, 8523.

[37] Cheng, Y.-J.; Luo, J.-D.; Hau, S.; Bale, D. H.; Kim, T.-D.; Shi, Z.-W.; Lao, D. B.; Tucker, N. M.; Tian, Y.-Q.; Dalton, L. R.; Reid, P. J.; Jen, A. K. Y. Chem. Mater. 2007, 19, 1154

[38] Yang, Y.-H.; Bo, S.-H.; Wang, H.-R.; Liu, F.-G.; Liu, J.-L.; Qiu, L.; Zhen, Z.; Liu, X.-H. Dyes Pigm. 2015, 122, 139.

[39] Wu, J.-Y.; Bo, S.-H.; Liu, J.-L.; Zhou, T.-T.; Xiao, H.-Y.; Qiu, L.; Zhen, Z.; Liu, X.-H. Chem. Commun. 2012, 48, 9637.

[40] (a) Wu, J.-Y.; Xiao, H.-Y.; Qiu, L.; Zhen, Z.; Liu, X.-H.; Bo, S.-H. RSC Adv. 2014, 4, 49737.

(b) Wu, J.-Y.; Peng, C.-C.; Xiao, H.-Y.; Bo, S.-H.; Qiu, L.; Zhen, Z.; Liu, X.-H. Dyes Pigm. 2014, 104, 15.

(c) Wu, J.-Y.; Liu, J.-L.; Zhou, T.-T.; Bo, S.-H.; Qiu, L.; Zhen, Z.; Liu, X.-H. RSC Adv. 2012, 2, 1416.

[41] Kim, T.-D.; Luo, J.-D.; Tian, Y.-Q.; Ka, J.-W.; Tucker, N. M.; Haller, M.; Kang, J.-W.; Jen, A. K. Y. Macromolecules 2006, 39, 6951.

[42] Zhang, H.; Huo, F.-Y.; Liu, F.-G.; Chen, Z.; Liu, J.-L.; Bo, S.-H.; Zhen, Z.; Qiu, L. RSC Adv. 2016, 6, 99743.

[43] Deng, G.-W.; Huang, H.-Y.; Peng, C.-C.; Zhang, A.-R.; Zhang, M.-L.; Bo, S.-H.; Liu, X.-H.; Zhen, Z.; Qiu, L. RSC Adv. 2014 4395.

[44] Li, Z.-A.; Wu, W.-B.; Hu, P.; Wu, X.-J.; Yu, G.; Liu, Y.-Q.; Ye, C.; Li, Z.; Qin, J.-G. Dyes Pigm. 2009, 81, 264.

[45] Li, Z.; Li, Q.-Q.; Qin, J.-G. Polym. Chem. 2011, 2, 2723. 